REDES- Revista hispana para el análisis de redes sociales

Vol.20,\#2, Junio 2011

http://revista-redes.rediris.es

\title{
De la Homofilia a la Cohesión social y viceversa ${ }^{1}$
}

Carlos Lozares y Joan Miquel Verd - QUIT, Departamento de Sociología² (UAB)

\section{Resumen}

Este artículo tiene cuatro objetivos articulados. Primero, presentar los contenidos con que, en la literatura ad hoc, se suelen identificar y analizar la Cohesión social y la Homofilia. Ambos conceptos provienen de tradiciones diferentes lo que origina definiciones no coincidentes. Segundo, encontrar un anclaje entre ambas concepciones que permita una conjugación de su identificación y tratamiento; se trata de las relaciones-interacciones entre individuos u otros agentes. Tercero, desarrollar una concepción abierta entre ambos contenidos, Cohesión social y Homofilia, a partir de un proceso social de retroalimentación entre ambos. Por fin, en cuarto lugar, presenta una posibilidad de convergencia como proceso de método y cálculo para estudiar dicha conjugación entre Cohesión social y Homofilia dentro de un proceso más amplio de retroalimentación entre ambas.

Palabras clave: capital social - cohesión social - homofilia - redes egocentradas

\begin{abstract}
This article has four articulated objectives. First, presenting the contents with which, in the ad hoc literature, Social Cohesion and Homofily are indentified and analysed. Both concepts steam from different traditions which have originated dissimilar definitions. Second, to find an anchorage among both conceptions that permits a conjugation in their identification and processing: it is a matter of the relations-interactions among individuals or other agents. Third, developing an open conception of both contents, Social Cohesion and Homofily, as a social process of mutual feedback. At last, in fourth place, the article presents a possibility of convergence as a process of method and calculation to study the conjugation between Social Cohesion and Homofily inside a more extensive process of feedback among both.
\end{abstract}

Key words: social capital - social cohesion - homophile - ego-networks

\footnotetext{
${ }^{1}$ Este artículo forma parte de la investigación cuya referencia es CSO2008-01470, subvencionada por el Ministerio de Educación y Ciencia en el marco del VI Plan Nacional de Investigación Científica, Desarrollo e Innovación Tecnológica 2008-2011. Los autores de este artículo pertenecen a dicho proyecto.

2 Enviar correspondencia a Carlos.lozares@uab.cat, Centre d'Estudis Sociològics sobre la Vida Quotidiana i el Treball (QUIT), Institut d'Estudis del Treball, (IET) Departament de Sociologia. Universitat Autònoma de Barcelona. 08193. Bellaterra. Cerdanyola del Vallès (Barcelona). Tel: 93.581.2405 / 93.581.1676. Fax: 93.581.2827.
} 


\section{Introducción}

La cuestión básica que se plantea en este artículo consiste en analizar hasta qué punto la Cohesión social es equiparable u homologable a la Homofilia social. Se trata de identificar los conceptos de Cohesión social y Homofilia a partir de sus respetivas tradiciones y examinar comparativamente sus contenidos. La propuesta que se hace en el artículo es que la Cohesión social y la Homofilia son procesos complementarios y partes de otro más envolvente. Para ello se examina un marco conceptual basado en el Capital social, Cohesión social y el Bonding junto a una metodología que asuma conceptos e indicadores propios a la teoría de Redes sociales (ver para esta temática en este mismo volumen Lozares et al. Cohesión, Vinculación e Integración sociales como formas de Capital social). El texto sigue la sucesión de estas temáticas.

\section{La Homofilia social}

La Homofilia se refiere al hecho social de que se den con mayor probabilidad prácticas, hábitos, opiniones, conocimientos mutuos y relaciones comunes entre individuos con características sociales iguales como edad, género, nivel educativo, ocupación, clase o estatus social, etc. que entre diferentes atributos. Esto es, que pares de individuos equivalentes en sus atributos sociales tienen más posibilidad de tener mayor frecuencia en sus encuentros, más concordancia en sus opiniones o prácticas similares que si no se da tal equivalencia. La mayor probabilidad de tales actividades o concordancias con los no similares o no equivalentes sería la Heterofilia (McPherson, Smith-Lovin y Cook, 2001).

En la literatura sobre Homofilia aparecen diversos criterios de clasificación. (i) Un primer criterio consiste en la distinción entre Homofilia individual y estructural. La homofilia individual se manifiesta o explica a partir de las preferencias individuales. En el criterio estructural las relaciones homofílicas están constreñidas por características, contextos o situaciones externas al individuo como por ejemplo la localización geográfica, ocupación, lugar de trabajo, etc. (ii) Este criterio distingue entre la Homofilia de elección y la inducida. Por la primera los individuos prefieren o deciden relacionarse, comportarse similarmente, etc. entre sí obedeciendo a la intención, voluntad o decisión de hacerlo; por la inducida, los individuos los comportamientos similares a sus semejantes se deben al contexto social o físico compartido o en el que están involucrados, por ejemplo el profesional. (iii) McPherson y otros (2001) introducen un nuevo criterio. Consideran dos tipos de Homofilia, la Homofilia de línea de base o de partida (baseline) que consiste en el 
hecho de que se de igualdad o equivalencia entre la proporción de presencia de una característica en un colectivo y la proporción de relaciones, comportamientos y creencias similares de un determinado tipo realizadas por dicho colectivo: si hay un $50 \%$ de mujeres en la población el 50\% de la mujeres van al cine: de alguna manera, la similitud en la proporción de comportamientos semejantes es 'porque toca'. De hecho, este tipo de Homofilia establece un punto de partida, una línea o nivel de flotamiento que marca el nivel de independencia entre el fenómeno de características sociales comunes y la similitud de comportamientos; el decir, indica el punto de partida de la razón social. Por otro lado, la Homofilia endogámica (inbreeding) 'vulnera' la lógica precedente de la Homofilia de base, al superar la proporción de comportamientos o relaciones similares al que corresponde a la proporción de la presencia de características sociales iguales en un colectivo. En esta homofilia pueden incluirse o al menos conjugarse también las de elección y, de alguna manera, la individualista. (iv) Otra distinción, entre Homofilia subjetiva frente a la objetiva, puede superponerse conceptualmente a las dualidades precedentes. La subjetiva tiene que ver con la percepción de la similitud o disimilitud en los atributos, lo que lleva a unos comportamientos homofílicos o heterofílicos, algo parecido a lo que más adelante llamaremos Homofilia CAT; por el contrario, la objetiva tiene que ver con que la realidad es similar o disimilar. (v) Otra connotación de la Homofilia va en otra dirección, es la que se establece entre Homofilia instrumental frente a la expresiva. Por la primera las afinidades son de carácter práctico como información, favor, consejo, apoyo... por la segunda lo son por contenidos emotivos. (vi) Lazarsfeld y Merton (1954) introdujeron la distinción entre Valor homofílico y Estatus homofílico. El Valor homofílico corresponde a la idea de que es más útil interactuar con los que mantienen valores similares (Ibarra 1993) que con los Alteri. Supone que la razón homofílica se explica menos directamente por características similares pues interviene como mecanismo una razón instrumental de utilidades que proviene de tal equivalencia en atributos.

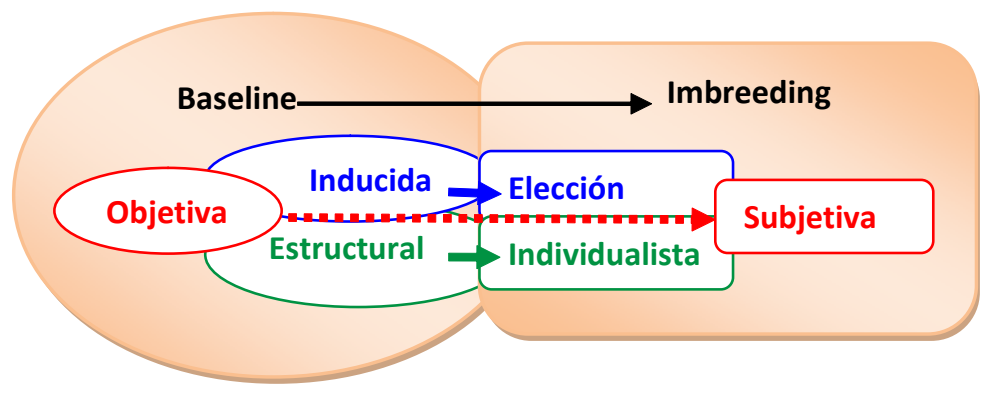

Gráfico 1. Connotaciones entre tipos de Homofilia 
Las connotaciones entre estos tipos de Homofilia se expresan en el Gráfico 1. No se ha de entender que todas las expresiones, de la izquierda o de la derecha sean asimilables o equivalentes en cada una de las partes; precisamente, en las definiciones dadas anteriormente se marcan diferencias, aunque sutiles. Con todo guardan un elevado grado de contagio en la connotación, más próximo cuanto más se concentren en el gráfico (Yuan y Gay 2006; Kossinets y Watts, 2009; Pearson, Steglich y Snijders 2006).

\section{Tendencias, desarrollo y cuestiones en los estudios sobre la Homofilia}

Los orígenes del concepto de Homofilia en la literatura sociológica se encuentran en Lazarsfeld y Merton (1954) con sus estudios sobre los procesos de amistad en los años 1950. Se basaron en los trabajos de Simmel (1971) y Park y Burgess (1921). Otros conceptos aparecen como sinónimos o casi-sinónimos a los de Homofilia versus Heterofilia, como son los de similitud frente a disimilitud, proximidad social frente a distancia, clausura social frente a apertura, homogeneidad frente a complementariedad. Algunos autores van más allá y hablan de valor y estatus homofílico y de similitud cognitiva (McPherson, Smith-Lovin y Cook 2001; Monge y Contractor 2003).

Los estudios iniciales se hacen sobre pequeños grupos sociales observando las relaciones entre sus miembros sobre todo en escuelas y vecindario. Wellman (1929) afirma que los alumnos tienen amistades y juegan en grupo o juntos en mayor proporción si tienen similares características socio-demográficas. Los atributos que abarcaron estos estudios suelen referirse a la edad, género, raza y educación (Loomis 1946) pero también a otras características sicológicas como inteligencia, actitudes y aspiraciones. Este tipo de estudios han continuado, por ejemplo, tomando la edad como criterio (Feld 1982), el género (Leenders 1996), la "raza" o etnicidad (Mollica, Gray y Trevino 2003), la educación (Marsden 1987). Han abundado, sobre todo en USA, los estudios sobre la discriminación racial en particular en escuelas, medios de transporte y lugares públicos. Otra tradición se ha centrado en los estudios sobre la influencia, positiva o negativa, de los grupos en el comportamiento de adolescentes. Monge y Contractor (2003) señalan dos líneas de investigación de la Homofilia dentro de la orientación sicosocial: la hipótesis de similitud atractiva (Byrne 1971) y la teoría de la autocategorización (Turner 1987). 
Entre los años 1970 y 1980 se da un salto metodológico en el estudio de la Homofilia al introducir la idea de redes-relaciones incluso con análisis de base muestral (Marsden, 1987), por ejemplo en estudios a gran escala en las escuelas (Duncan y otros 1972; Shrum y otros 1988), en otros colectivos (Laumann 1973, Fischer 1982) o en la población en general (Burt 1985; Marsden 1987). Estos estudios permiten examinar la Homofilia basándose en numerosas características (Blau 1977) pudiendo además dar una visión multidimensional del fenómeno homofílico.

\section{Algunas cuestiones sobre la Homofilia}

La identidad y tipos de estudios sobre las Homofilia y su capacidad de ser operativa y comprobable, no aclaran sus mecanismos explicativos, quedando como una caja negra inexplicada: por un lado existen características predefinidas $y$, por otro, los individuos que son semejantes en algunas de sus características tienen más posibilidad de comportamientos comunes, frecuencia de contactos o relaciones. No se explica el cómo y/o el porqué de este fenómeno de la Homofilia; esto es, la razón o los mecanismos sociales que lo generan.

Algunas de las razones explicativas de la Homofilia acostumbran a tener una base individual, sicológica y/o pragmática. El fenómeno homofílico simplifica los procesos de comunicación al poder predecir y evaluar comportamientos, (Festinger 1957; Werner y Parmelee 1979; Hamm 2000); ofrece un mayor sentimiento y atracción comunicativa al incrementar las expectativas de confianza, solidaridad, sentimiento cercanía (Portes y Sensenbrenner 1993; Mollica, Gary y Trevino 2003); tercero, reduce los riesgos asociados al trato o relaciones con gente diferente 0 no equivalente (Mouw 2003); y se da un coste menor en el mantenimiento de las relaciones homofílicas (Leenders 1996). En estos casos las no son las interacciones que generan a Homofilia; más bien es la Homofilia la que promueve las relaciones.

Para resolver el problema que hemos denominado de la caja negra más allá de los eslóganes tan gráficos de "similarity breeds connections" y "birds of a feather flock together" (McPherson y otros 2001) proponemos considerar la Homofilia parte de un proceso más general de vinculación retroalimentada entre Homofilia y Cohesión social tomando la interacción o relación social como mecanismo que da cuenta de tal composición. Ello permitirá abrir un tanto la caja negra evocada. 
La introducción de la connotación relacional-reticular

Los estudios homofílicos han estado tradicionalmente vinculados a características propias a factores o contextos demográficos como son la edad, etnicidad, etc.; sin embargo han ido apareciendo trabajos más focalizados sobre contextos organizativos vinculados a las redes sociales, por ejemplo, redes que conectan entidades como asociaciones, organizaciones, páginas web, etc.; estudios que se refieren a las organizaciones del trabajo (Burt 1992, 2000), a la fuerza del trabajo (Campbell 1988; Lin y otros 1981a, 1981b), o a los recursos interconectados necesarios para llevar a cabo tareas en el mundo de los negocios (Aldrich y otros 1996; Burt 1998).

Otras muchas investigaciones en Homofilia se centran en la homogeneidad o heterogeneidad de los vínculos o redes (Mcpherson y otros 2001; Ibarra 1995; Ibarra y Andrews 1993) como es el caso de los estudios de relaciones de matrimonio (Kalmijn 1998); amistad (Verbrugge 1983); trabajo (Ibarra 1995); contactos (Wellman 1996); conocimiento (Hampton y Wellman 2001); co-presencia en lugares públicos (Mayhew y otros 1995). También se han llevado a cabo investigaciones centradas en relaciones múltiples (Fischer 1982), los miembros tienden a tener más importancia cuanto más tipos de relaciones coexistan indicando así que el proceso homofílico es acumulativo.

Kossinets y Watts (2009) tratan la homofilia a partir de las relaciones o redes provenientes de interacciones, atributos y afiliaciones que transcurren en el tiempo. Esta conjunción permite comprobar que las relaciones 'apareadas' por similitud atributiva tienen más probabilidad que la media a tener relaciones. Muestran también que los cierres tríadicos pueden constreñir las oportunidades de nuevas relaciones y que los vínculos provienen de la conjunción de razones homofílicas de carácter estructural y de elección.

Sin embargo y a pesar de este cambio de rumbo que supone la introducción de las redes en el fenómeno homofílico, las relaciones-redes se siguen considerando como variable dependiente (McPherson y otros 2001) pues la causa se atribuye a la situación de los individuos en categorías similares. Es decir, no son las relacionesinteracciones las que producen las categorías sino que son éstas que generan las relaciones de afinidad. En este sentido la introducción de la redes no comporta un cambio importante en la consideración de la Homofilia. Con todo, se despeja un tanto la caja negra en la producción del fenómeno homofílico. 


\section{La Interacción, Relación, Cohesión social (CoS), Bonding y las redes}

Como señalábamos al principio del artículo, uno de sus objetivos consiste en identificar y contrastar los conceptos de Cohesión y Homofilia para considerarlos como componentes de un proceso más general. En este apartado nos centramos en la idea de Cohesión social, como en el precedente se ha hecho en la Homofilia.

\subsection{Las identidades sustantivas de la Cohesión social}

La Cohesión social de un colectivo, grupo, institución o sociedad se define habitualmente a partir del contenido sustantivo que se atribuye. Desde nuestra perspectiva el concepto de Cohesión social se ha de vincular también a la forma e intensidad en que se distribuyen las relaciones que se dan entre los miembros de un colectivo; más concretamente a sus relaciones Bonding, o relaciones hacia dentro o intra del colectivo. Por tanto, se parte también de una visión reticular o relacional de la Cohesión social sin dejar de lado, bien al contrario, que a las relaciones les son inherentes los contenidos sustantivos. Por tanto la Cohesión social pasa a ser la conjunción, primero, de un contenido determinado, por ejemplo de apoyo mutuo $\mathrm{y}$, segundo, de una forma $\mathrm{y} / \mathrm{o}$ intensidad relacional medible a partir de las relaciones Bonding.

Desde la psicología social se han enfatizado aspectos como actitudes, comportamientos, motivaciones, identidades, valores, etc. de los miembros de grupos como indicativos de Cohesión social. Moreno y Jennings (1937: 371) la definen como las "fuerzas que mantienen a los individuos dentro de los grupos de pertenencia"; Festinger (1950) y Back (1951) como campos de fuerzas que producen resultados sobre la constitución, mantenimiento y atracción de los miembros de un grupo; Gross y Martin (1952) insisten en que la Cohesión social es cuestión de actitudes de atracción a la personalidad de los miembros de un grupo operando sobre la decisión de permanecer o dejar el grupo. Estos contenidos de la Cohesión social siguen vigentes y no tienen por qué ser dejados de lado (McPherson y Smith-Lovin 2002). La Cohesión social ha ocupado un lugar central en la teoría de grupos dinámicos por su pertinencia con respecto a la constitución, permanencia o disolución de los grupos: los miembros de un grupo más cohesionado están más motivados a permanecer, participar y contribuir al bienestar del grupo. Estas aproximaciones sobre la identificación sustantiva de la Cohesión Social guardan un gran parangón con las razones apuntadas para el fenómeno homofílico. 
Otros autores dan a la Cohesión Social unos contenidos más sociológicos como son valores y propósitos comunes y participados, sentido de pertenencia y solidaridad social, ausencia de exclusión, redes y sentido de atracción hacia una identidad vinculada a un lugar o grupo (Forrest y Kearns 2001; Woolley 1998) inclusión, participación, reconocimiento y legitimidad (Jenson 1998).

Hay una cierta redundancia acumulativa en las definiciones sustantivas de la Cohesión social. Es claro que en una definición adecuada de la Cohesión social se han de incluir, y/o ser resultado de, mecanismos sociológicos y concretamente la interacción-relación porque no sólo posibilita la operacionalización de la Cohesión social sino porque es fuente de generar afinidades personales, influencia o frecuencia interactiva, y que por ende den pie a actitudes, representaciones, sentimientos compartidos y positivos en el mantenimiento del grupo (Berkowitz 1954; Festinger y otros 1950; Lott y Lott 1961). En nuestra visión la identidad e intensidad de la Cohesión social proviene de las interacciones-relaciones (redes) entre o dentro los miembros de un colectivo. La literatura sociológica genérica no una importancia suficiente a esta visión de la Cohesión social como composición forma y contenido. En el siguiente apartado profundizamos en ello (ver sobre la Cohesión social el artículo de Lozares et al. ya citado al principio).

\subsection{La Cohesión en perspectiva relacional, Bonding, y su medición}

\section{La Cohesión Social en perspectiva relacional-reticular}

Partimos de la idea básica de que la configuración de los grupos sociales y consecuentemente de los atributos y categorías sociales proviene de los fenómenos interactivos. Esto es, que las interacciones interpersonales, dinámicas de por sí, están en el principio de todo proceso social. Los resultados de las interacciones se distribuyen, una vez apropiados en redes más o menos cohesionadas, entre los individuos o agentes, originando grupos, atributos y categorías, esto es, estructura social. Estas relaciones intra, dentro de los colectivos, son relacionales internas o Bonding. Los Bondign están en la base de las estructuras de un colectivo pues las posiciones de los individuos en esta estructura origina los atributos. Por ello no es sorprendente, y es uno de los supuestos básicos de partida, que se dé una cierta equivalencia homológica entre los grupos cohesivos generados por las configuraciones reticulares y las posiciones 0 atributos estructurales que caracterizan los agentes. Los Bonding o relaciones intra generan posiciones estructurales en el colectivo que hace que su Cohesión social pueda diferir de unos colectivos a otros y ello además según los contenidos atribuidos a la Cohesión social 
como por ejemplo la confianza, apoyo, informativos, normas, valores, identidades etc. Así pues dentro de un colectivo las relaciones internas o Bonding hacen que sus agentes generen estatus o categorías en función de las distintas posiciones en la estructura de la red y por tanto configuraciones diversas de la Cohesión social (Shaw 1981). Este lado dinámico que es propio, desde nuestra perspectiva, a la Cohesión social no aparece en la perspectiva habitual con que se considera la Homofilia. Precisamente es esta dinámica interactiva la que posibilita establecer la complementariedad y la vinculación como un solo fenómeno de la Cohesión social y la Homofilia.

La Cohesión social se define pues por la composición o conjugación de un contendido sustantivo y de una configuración reticular que produce una estructura relacional entre individuos y/o grupos de un colectivo; su estructura e intensidad viene dada por la frecuencia, densidad y articulación de dichas relaciones. En este sentido, la Cohesión social permite, primero, tratarla con diferentes contenidos, como por ejemplo de apoyo mutuo, confianza o información $y$, segundo, considerarla desde un lado neutralidad a partir de su formalismo reticular lo que nos permite recurrir a los conceptos propios a la teoría de redes (Doreian y Fararo 1998; Granovetter 1973).

\section{La medición de la Cohesión social}

La componente formal Cohesión social basada en relaciones permite la apropiación de muchos conceptos e indicadores que provienen de la teoría de redes como componentes, reciprocidad, transitividad, densidad, grado medio, clausura, cliques, n-cliques, kores, alcanzabilidad, etc. Algunos de estos conceptos guardan una connotación directa con la idea de Bonding, otros, algo menor.

Burt $(2001,1997)$ sugiere la posibilidad de medir la Cohesión social de manera indirecta. Los 'agujeros estructurales' de un Ego tienen un mayor valor cuanto más sea su poder de intermediación con respecto a los Alteri relacionados directamente con el Ego. Esto es, el Bonding o Cohesión social, de un conjunto de nodos Alteri vinculados a un nodo Ego, medida pe. a partir de la densidad del conjunto, es tanto menor cuanto mayor sea el valor como 'agujero estructural' del Ego. Por tanto si el conjunto de los nodos de un colectivo tienen valores bajos como agujeros estructurales' tendrán un alto Bonding o Cohesión social. Precisamente por ello una muy alta densidad de contactos redundantes de relaciones fuertes y bajo valor de los 'agujeros estructurales' en un colectivo puede hacer disminuir la distribución y extensión de, por ejemplo, nuevas informaciones con colectivos externos (Burt 1992). Este conjunto de indicadores de la Cohesión social son propios o adecuados 
para las redes sociométricas. En este artículo no entramos en las llamadas relaciones o distribución del Capital social acuñada con la expresión de Bridging para la Vinculación social y de Linking para la Integración social pues nos centramos en la idea de Cohesión social, Bonding y Homofilia. La inicial alternativa bipolar Bonding y Bridging y la posterior trilogía introducida en la literatura de Capital Social (Woolcock 1998) de Bonding, Bridging y Linking como Integración social han dado mucho juego en la teoría del Capital Social. (ver artículos..) El hecho de que Granovetter (1973) afirme que la fuerza de los lazos, tanto en sentido formal por los Bonding o en contenidos como proximidad afectiva, apoyo, amistad, etc. de los miembros de un colectivo, procure altos niveles de Cohesión social a tal colectivo, no significa que dicho colectivo, como tal y/o como miembros del mismo, no mantenga relaciones con otros colectivos y/o sus miembros pe, ejemplo en relaciones débiles. Es decir que los Bridging y/o Linking coexisten con los Bonding. El conjunto es lo que configura la estructura del Capital social; en este sentido dichas estructura es una combinación de Cohesión, Vinculación e Integración social.

La tabla 1 muestra la oposición entre Bonding y Bridging por contenidos expresada por diferentes autores, aunque algunos de ellos no sean 'reticulistas' puros.

\begin{tabular}{|c|c|c|c|c|c|}
\hline Capital social & $\begin{array}{c}\text { Granovetter } \\
\text { (1973) } \\
\text { Henning } \\
\text { y Lieberd } \\
\mathbf{( 1 9 6 6 )}\end{array}$ & $\begin{array}{c}\text { Briggs } \\
\mathbf{( 1 9 9 8 )}\end{array}$ & $\begin{array}{c}\text { Woolcock } \\
\mathbf{( 1 9 9 8 )}\end{array}$ & $\begin{array}{c}\text { Gittel y Vidal } \\
\mathbf{( 1 9 9 8 ) ,} \\
\text { Putnam } \\
\mathbf{( 2 0 0 0 )}\end{array}$ & Lin (2001) \\
\hline Internalidad & Lazos fuertes & $\begin{array}{c}\text { Soporte } \\
\text { social }\end{array}$ & $\begin{array}{c}\text { Integración } \\
\text { (grupo) }\end{array}$ & $\begin{array}{c}\text { Capital } \\
\text { Bonding }\end{array}$ & $\begin{array}{c}\text { Acción expresiva } \\
\text { (lazos homofílicos) }\end{array}$ \\
\hline Externalidad & Lazos débiles & $\begin{array}{c}\text { Influencia } \\
\text { social }\end{array}$ & $\begin{array}{c}\text { Vínculo (entre } \\
\text { grupos) }\end{array}$ & $\begin{array}{c}\text { Capital } \\
\text { Bridging }\end{array}$ & $\begin{array}{c}\text { Acción instrumental } \\
\text { (lazos heterofílicos) }\end{array}$ \\
\hline
\end{tabular}

Tabla 1.Dos tipos de capital social (Kleinhans, Priemus y Engbersen 2007).

\section{La Homofilia social y la Cohesión social}

Este apartado está dedicado a especificar las diferencias entre Homofilia y la Cohesión pero también a encontrar afinidades y sobre todo a indagar líneas de convergencia. El resultado será el de considerarlas ambas como partes de un mismo proceso.

\subsection{Los procesos de la Homofilia y Cohesión social.}

Como hemos señalado, el recorrido conceptual de la Homofilia ha ido pasando de supuestos o concepciones sicosociales a inscribirse en una corriente más fructífera desde una perspectiva social como es la introducción de la interacción social y su 
correlato como relaciones. Ello además supone como se acaba de sugerir un enmarque teórico en el Capital social y metodológicamente en las Redes sociales. La opción con respecto a la Cohesión social es considerarla en perspectiva relacional-reticular bajo la forma de Bonding y con la cobertura teórica y aplicada de las Redes sociales sin menoscabo de sus contenidos. Por otro lado, con respecto a la Homofilia hemos visto que determinadas concepciones han ido introduciendo connotaciones relacionales sea como intermediación explicativa sea como consecuencias de la afinidad de atributos en una población o colectivo. Ello conduce a que la idea de considerar las interacciones-relaciones como nexo o eslabón entre una Cohesión social fundamentada en los Bonding y una visión de la Homofilia basada en vinculaciones atributivas; es decir, como base de una concepción más general que componga ambos procesos en uno solo. Para mejor conjugarlas se explicitan ambos procesos.

\section{El sentido del proceso de la Homofilia}

La objeción mayor al concepto de Homofilia proviene del posible una posible explicación tautológica, los resultados homofílicas en la población reproducen y refuerzan los atributos, que a su vez, etc.... Es decir, los atributos producen interacciones endógenas que redundan en una mayor constitución y/o confirmación de los grupos de partida. El resultado es que a partir de un atributo dado las interacciones consecuentes lo aseguran redundantemente sin una previsible posibilidad de cambio en dichos atributos y por tanto de la estructura social; lo que confiere a la Homofilia un cualidad autoreproductiva nada 'creacionista'. Si los atributos pre-existentes generan o determinan los mismos ¿cómo se generan los atributos $y$, sobre todo nuevos atributos?

La Homofilia va claramente de los atributos a las relaciones entre las personas dentro de las categorías a partir de las afinidades u otros mecanismos o razones mencionados, lo que choca con la importancia primordial de la relacionesinteracciones en procesos sociales de difusión, aprendizaje, imitación, socialización, distribución de recursos y también de los cambios. En esta unidireccionalidad de la Homofilia se agazapa y subyace un cierto determinismo de las categorías o atributos sobre la realidad interactiva (Grafico 2). La Homofilia no da cuenta de la caja negra sociológica en la producción de su propio efecto o bien recurre a pertinencias diferentes no estrictamente sociológicas aduciendo razones de predictibilidad, confortabilidad, cognitivas, que no dejas por ello de ser válidas... Desde luego, no hace intervenir directamente las relaciones e interacciones como mecanismos ya que son productos o resultados, cuando precisamente las 
interacciones están en el origen o generación de los atributos. En consecuencia no cuestiona el apriorismo explicativo otorgado a los atributos o categorías. Precisamente nuestro punto de vista consiste en que son las interacciones que hacen similares o diferentes a los individuos y así se generan los atributos y la estructura. En la lógica de la Homofilia los atributos se repetirían y redundantemente a sí mismos, entonces, ¿cuál son los mecanismos que los hacen cambiar en pertinencia social? La Homofilia va en una dirección (Gráfico 2) pero no da cuenta del camino inverso. Dicho de otra manera, dadas las relaciones e interacciones ¿cómo se generan los grupos o categorías? ¿hay razones para que también se de la heterofilia o los brigding o linking? Es claro que características similares socio-demográficas tienden a formar o tener pautas homogéneas en sus relaciones, pero el problema consiste en saber qué función tienen las interacciones como agencia social, pues son de por sí dinámicas creando además estructura como Bonding o Cohesión social.

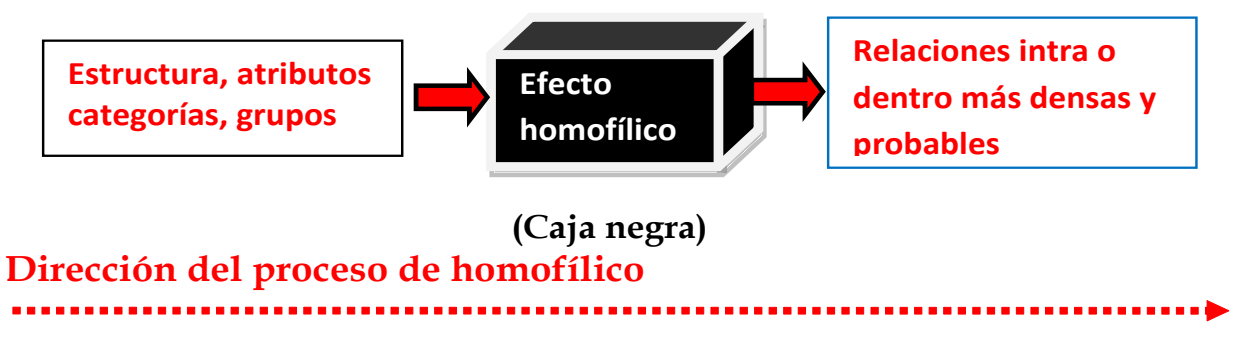

Gráfico 2. El proceso homofílico

En cuanto a la medición de la Homofilia, entendida sólo en su contenido clásico atributivo, sólo es cuestión de poner en parangón y analizar características de los individuos entre sí; es decir, hacer corresponder o asociar determinados atributos de los individuos con variables que expresen prácticas, hábitos, sentimientos, confianza, gustos, etc.

\section{El sentido del proceso de la Cohesión}

La Cohesión Social se enmarca en una perspectiva relacional o reticular a partir de los Bonding y, en cuanto a contenido, puede ser el de cualquier campo social. Como ya se ha señalado estructura los individuos o colectivos en posiciones y estatus, que, a su vez, originan características y atributos. Por tanto en la Cohesión Social se parte de un colectivo pero no necesariamente definido o delimitado por categorías o atributo sociales predeterminados, se parte de agentes con capacidad interactiva y/o relacional. Incluso la Cohesión Social no se vincula exclusivamente a lazos fuertes en el sentido pe. de confianza. De esta manera el concepto contiene 
un compuesto de neutralidad y transversalidad por su forma, lo que permite su medición, comparación y su extensión a cualquier contenido social.

Entre identificar primero los atributos y luego sus interacciones-relaciones consecuentes (se puede hablar de proceso causal en la Homofilia), la Cohesión Social parte de las interacciones-relaciones como origen y desarrollo del proceso. $Y$ es que la interacción (evidentemente en situación y contexto) es un mecanismo dinámico social suficiente como para dar cuenta de los resultados del los procesos sociales. En visión y pertinencia sociológica, y desde luego evolucionaria, lo primero son las relaciones-interacciones que, a su vez, generan las categorías. El proceso implícito a la Cohesión Social es la real caja negra pues en su desarrollo se generan los Bonding como resultados y a partir de ahí las estructura y los atributos (Gráfico 3)

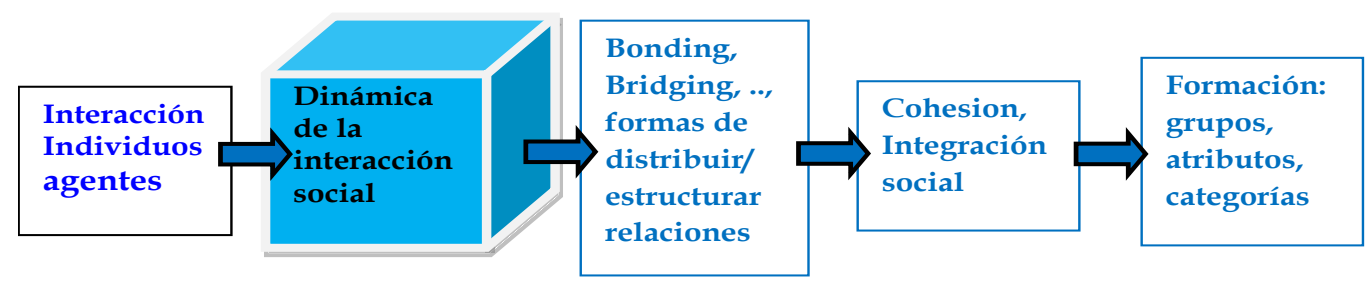

El proceso de cohesivo

Gráfico 3. El proceso cohesivo

En los procesos de Cohesión social no opera el cuasi-determinismo propio a la Homofilia, "iguales categorías producen más probablemente relaciones homofílicas dentro de su categoría" sino que se introduce un cierto grado de indeterminación. Sin embargo tampoco es válido afirmar que la interacción no está en parte determinada; hay resultados de recursos, atributivos en los agentes que provienen de procesos interactivos precedentes.

\subsection{La retroalimentación entre Cohesión social y Homofilia}

Burt (1982) introduce una reflexión de interés al hablar de dos sentidos de un proceso que analógicamente se pueden aplicar a la propuesta de la vinculación entre Cohesión social y Homofilia. El primer sentido es el que va de las normas comunes a los atributos similares y el segundo, inverso, el que los contactos comunes entre gente de los mismos atributos llevan a normas comunes (Burt 1982). El primero es el que da cuenta de la producción de los atributos; el segundo es el de un cierto determinismo estructural, esto es, que de atributos iguales se va 
a normas similares (Feld y Carter 1998). Esta consideración de Burt es interesante con respecto a nuestra propuesta de la visión retroalimentada de la Cohesión social y la Homofilia como de dos sentidos de una misma realidad; solamente se ha de cambiar normas por relaciones-interacciones, lo que también tiene sentido, puesto que las primeras se originan por las segundas.

Otra reflexión y distinción que viene a colación para abundar más en la propuesta de la retroalimentación de la Cohesión social y la Homofilia es la que hace White. Define una CaTegoría (CAT) como una agrupación de gente semejante en algún aspecto o desde algún punto de vista. Pero va más lejos de las clasificaciones clásicas de género, ocupación, religión, etnia o estilo de vida. Una CAT como tal contiene gente que además se reconocen como tal categoría (White 1965). Es decir, la CATegoría ha de ser adscrita y auto-reconocida. Además de tener características comunes referentes a cualquier atributo, género, clase social, etc. para un CAT se exige la condición de que se reconozcan sus características comunes y su participación en ellas. White $(1965,1992)$ vincula luego la noción de NET (red) con la de CAT (categoría) en el concepto de CATNET como un conjunto de individuos que comprenden categoría y redes. La idea de CAT puede ir en la línea de unificar la Homofilia, hay grupos o categorías estructurados en redes o relaciones, y la Cohesión social como reconocimiento relacional que puede ir de un mínimo de simple conocimiento a los de compartir y participar. En este sentido abunda en nuestra idea de retroalimentación (Rydgren 2005; Moody y White 2003).

La idea de Cohesión social y Homofilia retroalimentadas que introducimos consiste en un proceso abierto y retroalimentado entre atributos (características, grupos) e interacciones (relaciones-redes) que hace converger la Homofilia y la Cohesión social a partir de una dinámica helicoidal y progresiva. Veamos la descripción de este proceso de retroalimentación.

Hablar de interacciones, relaciones y estructura de la red es hablar de mecanismos y/o condiciones relacionales y de recursos que conducen a formas de distribución del Capital Social en formas Bonding, siendo la Cohesión social el resultado, lo que genera estructuras reticulares $y$, en consecuencia, nuevas o reproduce pretéritas identidades sociales o atributos. Es evidente que dichas categorías, a su vez, condicionan, como parte que son de la estructura relacional, la generación de interacciones como resultado; es la Homofilia. Y es que las categorías o atributos de pertenencia generados por relaciones previas se convierten en recursos estructurales de los sujetos para nuevas interacciones-relaciones, que son así 
condicionadas. El proceso de interacción-relación puede redundar así en el reforzamiento de las categorías previamente existentes o crear nuevas categorías; es decir, esta retroalimentación puede abundar en el mantenimiento de las categorías previas o producir nuevas dando pie a nuevas estructuras.

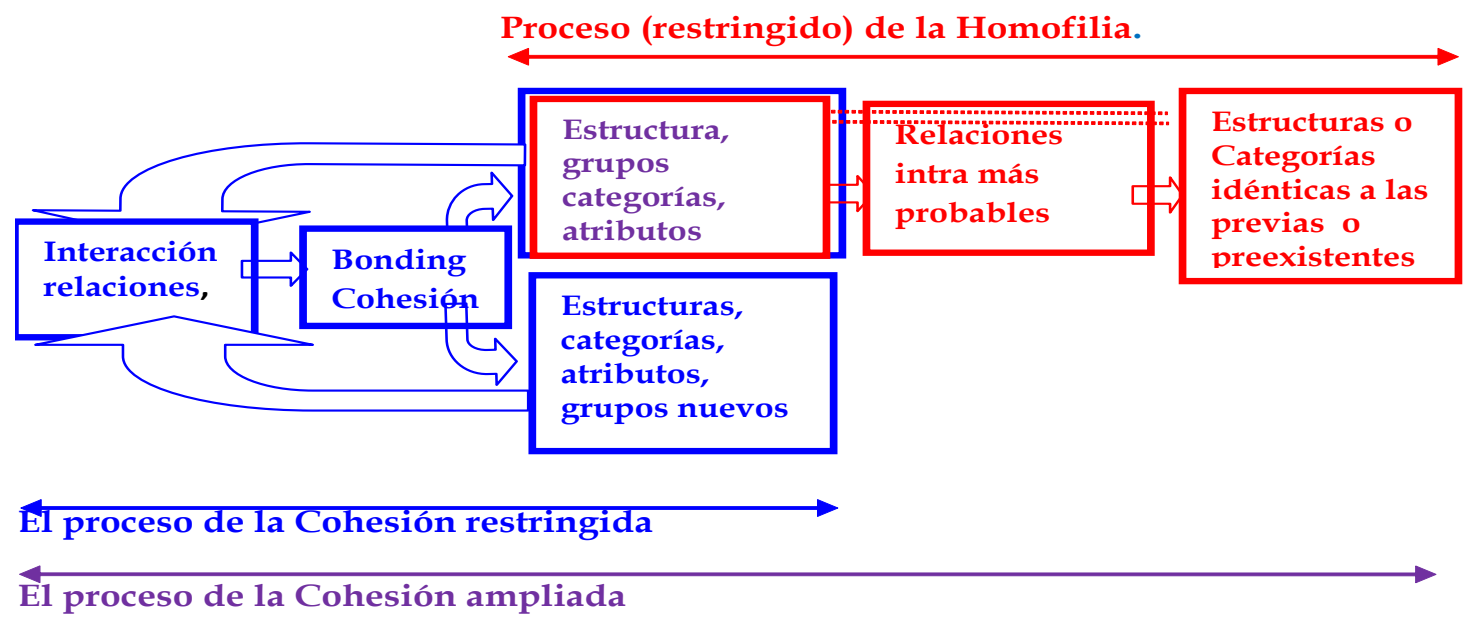

Gráfico 4. El proceso de retroalimentación

La Homofilia llena, al menos, una parte de todo este proceso: la que va de las categorías o atributos preexistentes a la generación de relaciones, que dado los resultados atribuidos a los proceso de Homofilia, contribuyen en alguna medida a mantener el estatus y estructura de atributos de partida (Gráfico 4).

Esta concepción retroalimentada sobre los vínculos entre Cohesión social y Homofilia abarca los dos sentidos del proceso, como corresponde a los fenómenos sociales reales. Así además se asegura su pertinencia sociológica y el juego de posibilidades de proceso global entre determinista y con ciertos grados de libertad, posibles como en todo proceso social. La Cohesión social y la Homofilia en su visión habitual pasan a ser complementarias como parte del proceso. La Homofilia es más proclive a los efectos o resultados redundantes o predeterminados: los miembros que se parecen interaccionan más (es el lado clásico homofílico). Pero precisamente los que más se parecen son porque interaccionan más (es el lado clásico cohesivo). El proceso en su conjunto queda abierto pero el centro ya no son la Cohesión social - la Homofilia sino la relación-interacción entre agentes. Es precisamente por ello que el sistema social mantiene su caracterización como atributos, está en proceso de transición o los cambia; hay posibilidad de una alternativa a la diferenciación social existente. Estas reflexiones tienen también interés para hacer pasar de un modelo basado en características o variables atributivas a otro de naturaliza relacional-reticular. 


\section{Reflexiones a modo de conclusión}

Las reflexiones y propuestas hechas en este artículo provienen y se refieren a preguntas y discusiones en el proceso de una investigación en la que por primera vez nos enfrentábamos a un tratamiento conceptual y metodológico de redes egocentradas para muchos Egos. La investigación está centrada en le idea del Capital social y en su distribución en formas de Cohesión social entre los Egos y los Alteri. Dentro del proceso de investigación se ha ido introduciendo la idea de Homofilia como un concepto próximo, al menos en una primera aproximación, al de Cohesión social manejado en la investigación. La pregunta que surge es evidente, ¿qué es lo estábamos calculando, la Homofilia o la Cohesión social? La respuesta a esta pregunta ha llegado a partir de dos consideraciones. La primera es la introducción de la perspectiva relacional-interactiva en la consideración de ambos conceptos junto a la idea de Bonding como forma de distribución del Capital social en la Cohesión social; la segunda, las posibilidades que ofrece la conjugación de dos métodos en el tratamiento de las redes, por un lado, los centrados en las relaciones de los Egos con los Alteri con sus alteri por un tratamiento atributivo en la línea de la Homofilia social y, por otro, los centrados en las redes sociométricas en la línea de la Cohesión reticular. Ello nos ha conducido, primero a clarificar los conceptos de Cohesión social y de Homofilia, segundo a examinar sus vínculos y, tercero a tratar de construir un modelo de retroalimentación mutua entre ambas como en un bucle progresivo, más allá incluso de entenderlas como dos caras de un misma moneda que es una visión más estática. Esta indagación no es pues el resultado de un planteamiento teórico inicial, más bien, el problema teórico ha ido surgiendo como resultado de un proceso de investigación al que se hemos tratado de dar solución.

\section{Bibliografía}

Alahyane, Mohamed (1986). "Stratégie matrimoniale et mariage à Lakhsas". BESM, no 158, Rabat.

Altorki, Soraya (1986). Women in Saudi Arabia: Ideology and Behavior among the Elite. New York: Columbia University Press.

Bonte, Pierre y Conte, E. (1991). "La tribu arabe. Approches anthropologiques et orientalistes" en Al-Ansab. La quete des origines.Anthropologie historique de la société tribale arabe. Louvaine: Ed. de la Maison des Sciences de I'Homme Paris, pp. 13-48. 
Aldrich, H.E.; Elam A.; Reese P.R. (1996). "Strong ties, weak ties and strangers: Do women business owners differ from men in their use of networking to obtain assistance?", en Birley S.; MacMillan I. (ed), Entrepreneurship in a Global Context. London: Routledge, pp. 1-25.

Back, K.W. (1951). "Influence through social communication". J. Abnorm. Soc. Psych., 46:9-23.

Berkowitz, L. (1954). "Group standard, cohesiveness, and productivity", Hum. Relat., 7:509-19.

Blau, P.M. (1977). Inequality and Heterogeneity. New York: Free Press.

Borgatti, S.P.; Everett M.G.; Shirley P.R. (1990). "LS sets, lambda sets and other cohesive subsets", Soc. Networks, 12:337-57.

Bourdieu, P. (1986). The Forms of Capital, Handbook of Theory and Research for the Sociology of Education. New York: Greenwood Press.

Burt, R. S. (1997). "A note on social capital and network content", Social Networks, $19(4): 355-373$.

Burt, R. S. (2001). "Structural holes versus network closure as social capital", en Lin N.; Cook K.; Burt R.S (Eds.), Social Capital: Theory and Research. New York: Aldine de Gruyter, pp. 31-56.

Burt, R.S. (1992). Structural holes: the social structure of competition. Cambridge: Harvard University Press.

Burt, R.S. (1998). "The gender of social capital", Rationality Soc. 10:5-47.

Burt, R.S. (2000). "The Network Structure of Social Capital", en Robert I. Sutton y Barry M. Staw (eds.), Research in Organizational Behavior, Greenwich, CT: JAI Press.

Byrne, D. E. (1971). The Attraction Paradigm. New York: Academic Press.

Campbell, K.E. (1988). "Gender differences in job-related networks", Work Occup. 15: $179-200$.

Doreian, P.; Fararo T. (eds.) (1998). The Problem of Solidarity: Theories and Models. Amsterdam, Neth.: Gordon \& Breach. 
Duncan, O.D.; Featherman D.L; Duncan B. (1972). Sociometric Background and Achievement, New York: Seminar.

Feld, Scott L. (1982). "Structural Determinants of Similarity among Associates", American Sociological Review, 47: 797-801.

Festinger, L. (1957). A Theory of Cognitive Dissonance. Stanford, Calif.: Stanford University Press.

Festinger, L.; Schachter S.; Back K.W. (1950). "Social Pressures in Informal Groups: A Study", Annu. Rev. Sociol., 30: 409-425.

Fischer, C.S. (1982). To Dwell Among Friends: Personal Networks in Town and City. Chicago: University of Chicago Press.

Forrest, R.; Kearns A. (2001). "Social cohesion, social capital and the neighbourhood", Urban Studies, 38(12): 2125-2143.

Friedkin, N.E. (2004). "Social cohesion", Annu. Rev. Sociol., 30: 409-25.

Granovetter, M.S. (1973). "The strength of weak ties", Am. J. Sociol., 78: 1360-80.

Gross, N.; Martin W.E. (1952)." On group cohesiveness", Am. J. Sociol., 57: 54654.

Halpern, D. (2005). Social Capital. Cambridge: Polity Press Cambridge.

Hamm, J.V. (2000). "Do Birds of a Feather Flock Together? Individual, Contextual, and Relationship Bases for African American, Asian American, and European American Adolescents' Selection of Similar Friends", Developmental Psychology, 36 (2):209-19.

Hampton, K.N.; Wellman B. (2000). "Examining community in the digital neighborhood: Early results from Canada's wired suburb". en Isbister K.; Ishida T. (eds.) Digital Cities: Technologies, Experiences and Future Perspectives, Heidelberg: Springer-Verlag, pp.194-208.

Hawkins, R.L.; Maurer, K. (2009). "Bonding, Bridging and Linking: How Social Capital Operated in New Orleans following Hurricane Katrina", British Journal of Social Work 40(6): 1777-1793.

Ibarra, H., (1995) "Race, opportunity, and diversity of social circles in managerial networks", Academy of Management Journal, 38 (3): 673-703. 
Ibarra, H.; Andrews S. B. (1993). "Power, social influence, and sense making: Effects of network centrality and proximity on employee perceptions". Administrative Science Quarterly, 38 (2): 277-303.

Jenson, J. (1998). "Mapping Social Cohesion: The State of Canadian Research", Canadian Policy Research Network (CPRN), Study No. F|03, Ottawa, Canada.

Kalmijn, M. (1998)."Intermarriage and homogamy: causes, patterns and trends", Annu.Rev. Sociol. 24:395-421

Kleinhans, E.R.; Priemus H.; Engbersen G. (2007). "Understanding Social Capital in Recently Restructured Urban Neighbourhoods: Two Case Studies in Rotterdam", Urban Studies, 44(5 y 6): 1069-1090.

Kossinets, G.; Watts D.J. (2009). "Origins of Homophily in an Evolving Social Network", AJS, 115(2): 405-50

Laumann, E.O. (1973). Bonds of Pluralism: The Form and Substance of Urban Social Networks. New York: Wiley.

Lazarsfeld, P. F.; Merton R.K. (1954). "Friendship as a social process: A substantive and methodological analysis", en Berger M.; Abel T.; Page C.H (eds.), Freedom and Control in Modern Society, New York: Octagon Books, pp.18-66.

Leenders, R. T. (1996). "Evolution of friendship and best friendship choices", Journal of Mathematical Sociology, 21 (1-2): 133-148.

Lin, N.; Ensel N.; Vaughn J.C. (1981a). "Social resources and strength of ties: structural factors in occupational status attainment". Am.Sociol. Rev., 46: 393-405.

Lin, N.; Ensel W.M.; Vaughn J.C. (1981b). "Social resources and occupational status attainment", Soc. Forces, 59:1163-81

Loomis, C.P. (1946). "Political and occupational cleavages in a Hanoverian village", Sociometry, 9: 316-33.

Lott, A.J.; Lott B.E. (1961). "Group cohesiveness, communication level, and conformity", J. Abnorm. Soc. Psych., 61:408-12.

Louch, H. (2000). "Personal network integration: transitivity and homophily in strong-tie relations". Social Networks, 22: 45-64. 
Lozares, Carlos; Verd, Joan Miquel; López-Roldán, Pedro; Martí, Joel y José Luis Molina (2011). "Cohesión, Vinculación e Integración sociales como formas de Capital social", REDES-Revista hispana para el análisis de redes sociales, vol. 20 \#1 (http://revista-redes.rediris.es)

Marsden, P.V. (1987). "Core Discussion Networks of mericans." American Sociological Review, 52:122-313.

Mayhew, B.H. (1980). "Structuralism versus Individualism: Part 1, Shadowboxing in the Dark." Social Forces, 59 (2): 335-75.

McPherson, J.M.; Smith-Lovin, L.; Cook, J. (2001). "Birds of a feather: homophily in social networks", Annual Review of Sociology, 27:415-444

McPherson, M.; Smith-Lovin L. (2002). "Cohesion and membership duration: linking groups, relations and individuals in an ecology of affiliation", Adv. Group Process, 19:1-36

Monge, P. R.; Contractor, N. (2003). Theories of Communication Networks. Oxford: Oxford University Press.

Monica, K.; Gray, B.; Trevino K. (2003). "Racial homophily and its persistence in newcomers' social networks", Organization Science, 14(2):123-136.

Moody, J.; White D.R. (2003). "Structural cohesion and embeddedness: a hierarchical concept of social groups", Am. Sociol. Rev. 68:103-27.

Moreno, J.; Jennings H. (1937). "Statistics of social configurations". Sociometry, $1: 342-74$.

Mouw, T. (2003). "Social Capital and Finding a Job: Do Contacts matter?", American Sociological Review, 68:868-98.

Park, R.E.; Burgess E.W. (1921). Introduction to the Science of Sociology. Chicago: Univ. Chicago Press.

Pearson, M.; Steglich Ch.; Snijders T. (2006). "Homophily and assimilation among sport-active adolescent substance users", Connections 27(1), 47-63

Portes, A. (1998). 'Social capital: Its origins and applications in modern sociology', Annual Review of Sociology, 24: 1-24. 
Portes, A.; Sensenbrenner, J. (1993). "Embeddedness and Immigration: Notes on the Social Determinants of Economic Action." American Journal of Sociology, 98:1320-50.

Putnam, R. D. (1993) Making Democracy Work: Civic Tradition in Modern Italy. Princeton, NJ: Princeton University Press.

Putnam, R. D. (2000). Bowling Alone: The Collapse and Revival of American Community. New York: Simon \& Schuster

Rydgren, J. (2005). "Bridging Different Worlds?" Economy, Politics and Brokerage Roles in Sweden", Acta sociologica, 48: 117

Shaw, M.E. (1981). Group Dynamics: The Psychology of Small Group Behavior. NewYork: McGraw-Hill.

Shrum, W.; Cheek N.H., Hunter S.M. (1988). "Friendship in school: gender and racial homophily", Soc. Educ., 61:227-39

Simmel, G. ([1908] 1971), "The Stranger", en Levine D. (ed.), Simmel on individuality and social forms: Selected writings. Chicago: University of Chicago Press, pp. 43-49.

Turner, J. C. (1987). Rediscovering the Social Group: A Self-Categorization Theory. Oxford: Basil Blackwell.

Verbrugge, L.M. (1983). "A research note on adult friendship contact: a dyadic perspective". Soc. Forces, 62: 78-83.

Wellman, B. (1929). "The school child's choice of companions", J. Educ. Res., $14: 126-32$.

Wellman, B. (1996). "Are personal communities local? A Dumptarian reconsideration". Soc. Networks, 18:347-54.

Werner, C.; Parmelee P. (1979). "Similarity of Activity Preferences among Friends: Those Who Play Together Stay Together Social", Psychology Quarterly, 42(1):6266.

White, H. C. (1965). "Notes on the Constituents of Social Structure". Mimeo. Department of Sociology, Harvard University. 
White, H. C. (1992). Identity and Control: a Structural Theory of Social Action, Princeton, NJ: Princeton University Press.

Woolcock, M. (1998). "Social capital and economic development: towards a theoretical synthesis and policy framework", Theory and Society, 27: 151-208.

Woolley, F. (1998). "Social Cohesion and Voluntary Activity: Making Connections" Paper presented at the CSLS Conference on the State of Living and the Quality of Life of Canada. Ottawa Canada, October 30-31, 1998,

Yuan, Y. C.; Gay, G. (2006). "Homophily of network ties and bonding and bridging. Social capital in computer-mediated distributed teams", Journal of ComputerMediated Communication, 11(4), article 9. 